\title{
The Design of Mine Encapsulated and Intrinsically Safe Explosion-proof Computers
}

\author{
GAO Jun ${ }^{1, *}$ \\ ${ }^{1}$ CCTEG XI'AN Research Institute, Xi'an, Shaanxi, 710077, China
}

\begin{abstract}
With the continuous improvement of the level of intelligence in coal mines, construction sites have put forward new requirements for the performance and compatibility of mining computers. Aiming at the field conditions of intelligent underground drilling in coal mines, this paper designs a mine-used encapsulated and intrinsically safe computer as a data processing terminal, with the characteristics of a large display screen, a moderate overall weight, powerful operating performance and multiple compatible interfaces, which can replace the explosion-proof and intrinsically safe computers in current field applications.
\end{abstract}

\section{Introduction}

Mine explosion-proof computers are mainly used for data reception, processing, display and storage of various monitoring and control systems in coal mines, and are also a platform for human-computer interaction. Due to the explosion-proof requirements of underground electrical equipment in coal mines, daily use computers can't be directly connected to underground use in coal mines. Explosion-proof design is required to meet the requirements for explosion-proof underground in coal mines.

At present, explosion-proof computers or similar data processing terminals in coal mines are mainly explosionproof and intrinsically safe or intrinsically safe, with powerful performance and many compatible interfaces, large size of the explosion-proof shell, heavy weight, high power consumption and limited in installation and use space, while intrinsically safe computer products have low power consumption, small size, and light weight, with limited performance and compatible interfaces.

According to the requirements of intelligent underground drilling in coal mines, this paper designs a mine encapsulated and intrinsically safe computer suitable for intelligent underground drilling in coal mines. It has the advantages of large display size, moderate overall weight, powerful operating performance and multiple compatible interfaces.

\section{Encapsulated and Intrinsically Safe Explosion-proof Computers}

The explosion-proof computer designed in this paper is a universal explosion-proof computer compatible with multiple interfaces suitable for underground coal mines. As the upper computer of the intelligent drilling equipment, it can complete the bottom data collection and processing, and carry out human-computer interaction. It adopts an encapsulated and intrinsically safe explosionproof type, with specific electrical block diagram shown in Figure 1, including encapsulated power supply components, computer motherboards, interface isolation panels, LCD screens, touch control panels, WIFI/Bluetooth modules, and mine wireless explosionproof USB flash drive and mining explosion-proof keyboard. Encapsulated power supply components, computer motherboard, interface isolation board, LCD display, touch control panel and WIFI/Bluetooth module are packaged in a protective shell to form a host. The encapsulated power supply component provides threeway isolated intrinsically safe power supply for the computer, and the back-end circuits are isolated or independent of each other. The computer is equipped with a touch control panel installed on the outer surface of the LCD screen, by using the infrared positioning principle to control the computer through finger touch. The $12 \mathrm{~V}$ intrinsically safe input for backlight power supply in the LCD screen and the signal-controlled LVDS interface are independent of each other as two independent circuits.

The computer is equipped with many peripheral communication interfaces, which are connected to external devices through the interface isolation board, including: RS232, 485, Ethernet, CAN, USB, WIFI and Bluetooth. It can also be compatible with design and custom communication interfaces to meet most production and application sites. In the interface isolation board, the circuits before and after isolation are separately powered, so as to ensure that the output terminal and the main board circuit are isolated from each other. Except for the encapsulated power components in the computer, the rest of the circuits are intrinsically safe circuits. The computer can realize external operation and control through mining explosion-proof keyboard and touch control panel.

The computer is equipped with a wireless USB flash drive for mining, which can communicate with the host

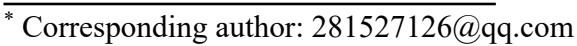


through wireless WIFI, access data, improve the stability of the USB flash drive application, and avoid long-term plug-in and unplug the communication interface causing unstable communication interface. The invention has strong practicability, low power consumption, compact structure, reduced volume and mass relative to explosionproof computers, improved on-site adaptability, which is suitable for use as an upper computer data interactive processing device in a coal mine working environment.

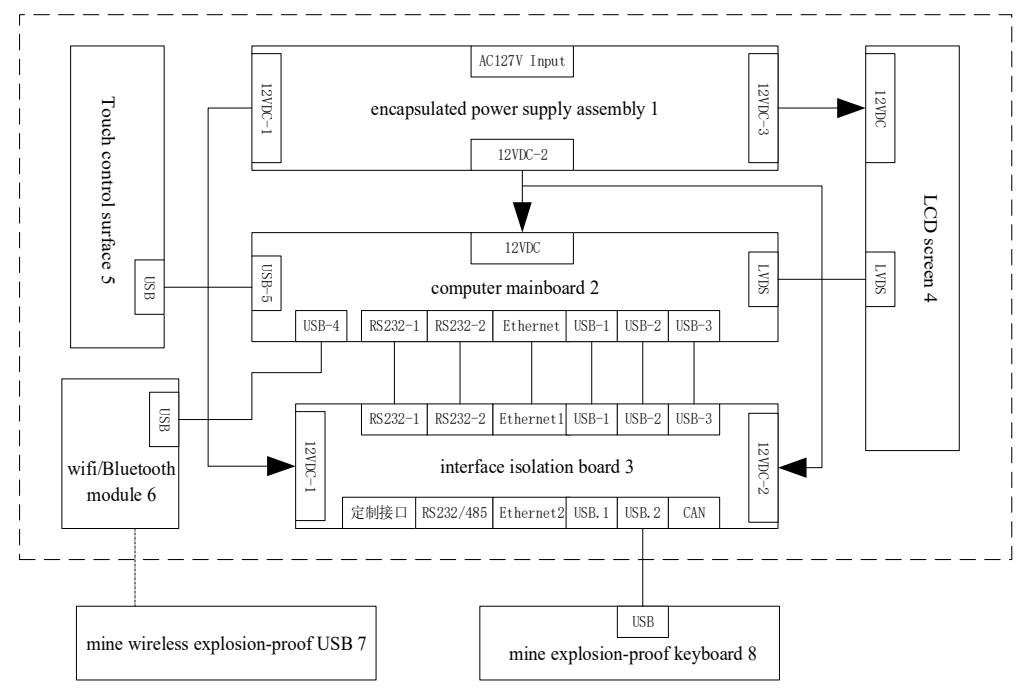

Fig. 1. Overall electrical schematic diagram of computer

\subsection{Encapsulated Power Supply}

The encapsulated power supply introduces external AC power and converts it into three-channel DC power for internal power supply, with internal circuit block diagram shown in Figure 2. The circuit as a whole is encapsulated in accordance with GB 3836.9-2010 encapsulation type requirements, including an $\mathrm{AC} 127 \mathrm{~V}$ to $\mathrm{DC} 12 \mathrm{~V}$ isolation Power module, three DC12V to DC12V isolation modules and three matching dual voltage limiting current limiting protection circuits. The encapsulated power supply component is powered by $127 \mathrm{~V}$ alternating current, which is converted into $12-24 \mathrm{~V}$ direct current through ACDC isolated power supply 1 , which supplies power to the back-end DCDC isolated power supplies 2, 3, and 4, and the isolated power supplies 2, 3, and 4 output $12 \mathrm{~V}$ direct current stably. After double voltage and current protection 6,7 , and 8 , it is converted into $12 \mathrm{~V}$ intrinsically safe power output. According to different load requirements, the overcurrent protection value of the three intrinsically safe power supplies is different. Among them, the load transmission for the front-end power supply of the main board and the isolation board, the overcurrent protection value can also be passed, with the overcurrent protection level at 2A. One way supplies power for the LCD signal display with the overcurrent protection value at $1.15 \mathrm{~A}$, the other way is supplies power for the back end of the isolation board and external intrinsically safe equipment, with small load and the overcurrent protection value at $0.8 \mathrm{~A}$.

The encapsulating material uses the HT6302 encapsulant of Shanghai Huitian New Chemical Materials Co., Ltd., which belongs to epoxy resin glue, with the temperature range at $-40 \sim 90^{\circ} \mathrm{C}$. During the encapsulation process, the circuit board is connected to the input and output cables and placed in the protective shell for integral encapsulation, with the thickness of the encapsulation less than $2 \mathrm{~mm}$. The encapsulated power input cable is a nonsafety terminal. The encapsulated cable can withstand the pulling force required by explosion-proof without damage.

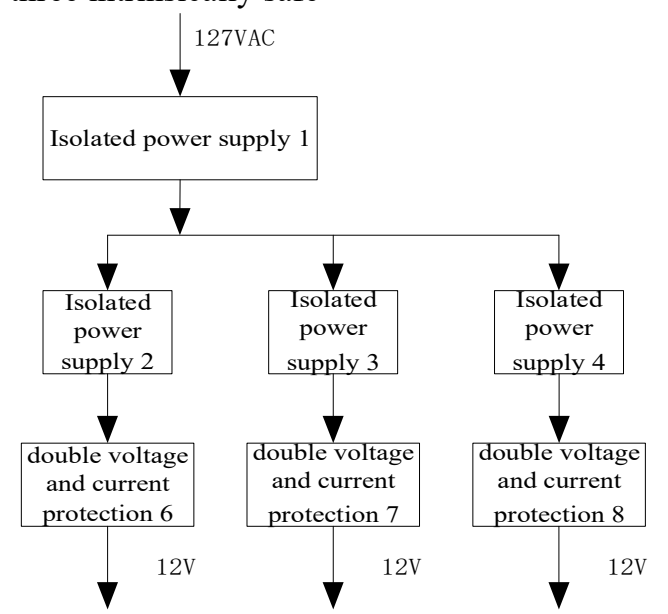

Fig. 2. Electrical block diagram of encapsulated power supply 


\subsection{Mechanical Structure}

The various peripheral interfaces (including: USB, RS232/485, RJ45, CAN and other non-standard communication interfaces) of the computer for data interaction must be isolated before they can communicate with other devices. According to the requirements of GB3836.4-2010, the isolation strength shall not be lower than 500VAC.

USB isolation uses ADI's iCoupler ${ }^{\circledR}$ technology USB port isolator, which combines high-speed CMOS technology with monolithic air-core transformer technology to provide excellent working performance and is easy to integrate with low-speed and full-speed USB compatible peripherals. The USB implemented by many microcontrollers only provides $\mathrm{D}+$ and $\mathrm{D}-$ lines to the external pins, and the power supply terminal is separated by DCDC. The RS232/485 isolation scheme uses ADI's ADM3251E isolation chip that does not require a separate isolated DC-DC converter, which can provide an overall isolation solution with a data rate of up to $460 \mathrm{kbps}$ and a rated temperature range of $-40^{\circ} \mathrm{C}$ to $+85^{\circ} \mathrm{C}$. The network port isolation adopts the S16-1102 transformer module packaged by the module for isolation. CAN isolation adopts the USB to CAN module LCUSB-131AX, which realizes the expansion of the computer interface and the isolation of the CAN interface at the same time.

\subsection{Supporting wireless USB}

In order to meet the simple and beautiful appearance design requirements of the computer, the mechanical structure of the computer is shown in Figure 3. The display window on the computer is composed of three layers. The uppermost is an infrared touch control panel, the middle is $5 \mathrm{~mm}$ thick tempered glass, and the lowermost is LCD. The touch control panel is a glass panel that is basically the same size as the window LCD screen, but there is a infrared signal transmitter and receiver in the periphery, which can be installed on the outermost surface to receive the infrared touch signal on the surface. Then it can be changed into an electrical signal and sent to the computer motherboard for processing. Tempered glass installed in the middle can not only withstand the pressure exerted on the touch panel on the outer surface, but also facilitate the protective cooperation with other parts of the shell. The liquid crystal display is installed under the toughened glass, that is, inside the protective shell, which has good protection, high safety, and better stability, and does not affect the display effect.

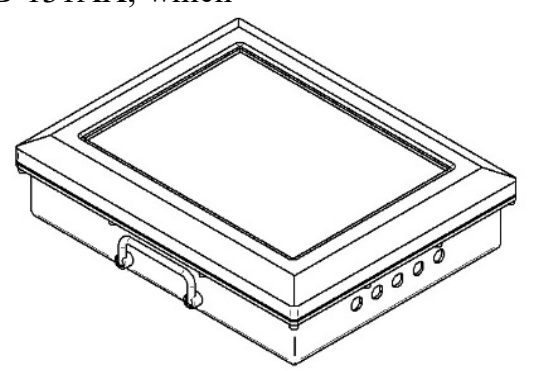

(a) Computer overall axial diagram

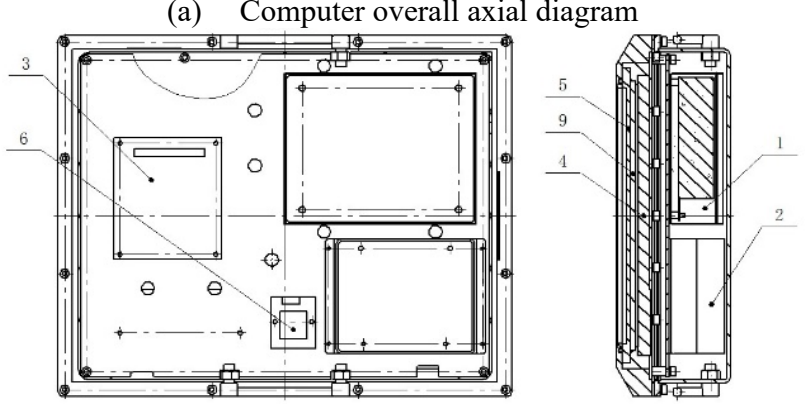

(b) Location map of key computer components

Fig. 3. Explosion-proof computer structure diagram

\subsection{Supporting Wireless USB Flash Drive}

The computer-equipped wireless USB flash drive for mine is an important part of perfecting the performance of the explosion-proof computer. In the case that some drilling sites do not have the connection to the underground ring network, the data transfer between the underground and the ground can be realized. The electrical block diagram is shown in Figure 4. It consists of five parts, that is, lithium battery, dual protection circuit, main control module, storage module and wireless communication module. It can communicate with explosion-proof computers through wireless (WIFI) or wired (USB). The lithium battery is used as the energy-consuming storage unit of the wireless USB flash drive for mining. After being charged with a special charger on the ground, it is used in the coal mine to supply power for the back-end working module. When discharging a lithium battery, it first needs to pass through the dual current protection circuit for current limiting (because it is battery-powered output, the voltage output value itself has an upper limit, so there is no need to limit the voltage), to ensure that the dual current protection circuit output is intrinsically safe. And then it supplies power to the back-end main control module, wireless communication module and storage module. The main control module communicates with the storage module through the parallel port to increase the speed of 
reading and writing. The data can be transmitted and received by communication with the wireless communication module. It can also directly communicate with explosion-proof computing through the USB interface. It can also communicate with explosion-proof computers through WIFI wireless.

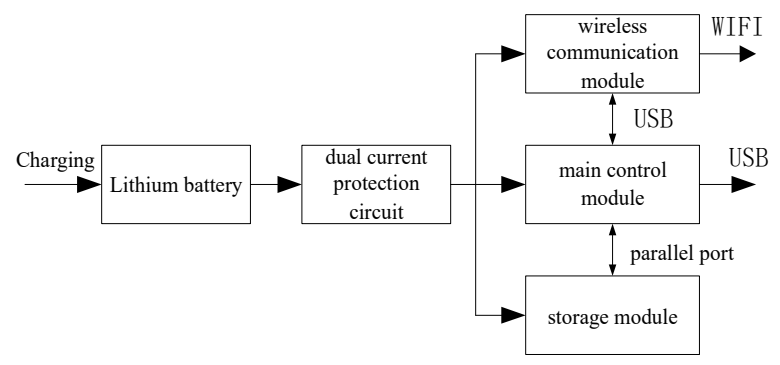

Fig. 4. Electrical block diagram of mine wireless USB

\section{Conclusion}

With the continuous improvement of the level of intelligence in coal mines, more and more electrical systems such as measurement, calculation and execution used in on-site construction, and the requirements for data terminals will be more and more subdivided. At the same time, due to the particularity of the construction environment, mining electrical equipment must meet explosion-proof requirements in order to avoid safety accidents, but explosion-proof requirements have many limitations on the design of instrument performance. In order to meet the actual needs of on-site working conditions and ensure safe production operations, various explosion-proof methods must be used flexibly to meet the requirements of instrument design under the trend of the new era.

\section{Funded Projects}

[1] Development of Explosion-proof Turbine Generator for Underground Wireless While Drilling Measurement (2018XAYMS01).

[2] Major National Science and Technology Special Tasks in the 13th Five-Year Plan

(2016ZX05045-003-001).

[3] Key R \& D plan of Shaanxi Province General items (2021GY-331).

\section{References}

1. DENG Nan, ZHAO En-biao, ZHENG Wan-bo,Data Acquisition Integration Mine Intrinsically Safe Computer Design,Coal Mine Machinery,2016, 37(3)

2. SHAN Chengwei, Mine belt conveyor control system based on mine intrinsically safe computer, Coal Mine Modernization,2013, (6)

3. QIAN Shulin, WANG Xuewu, Design of Signal Isolator for Explosion-proof and Intrinsic Safety Computer Used in Coal Mine, Industry and Mine Automation, 2012, 38(4) 